\title{
Vigorexia, um distúrbio alimentar na modernidade
}

\author{
Vigorexia, an eating disorder in modern times \\ Vigorexia, un transtorno alimentario em los tiempos modernos
}

Recebido: 08/04/2021 | Revisado: 15/04/2021 | Aceito: 17/04/2021 | Publicado: 02/05/2021

Cely Araújo dos Santos

ORCID: https://orcid.org/0000-0003-3089-882X Faculdade de Comunicação, Tecnologia e Turismo de Olinda, Brasil

E-mail: celysntos@hotmail.com

Gleyce Kelly de Araujo Bezerra

ORCID: https://orcid.org/0000-0002-9388-7389

Universidade Federal de Pernambuco, Brasil

E-mail: gleycearaujo.nutri@gmail.com

Maria Suzane da Silva Barbosa

ORCID: https://orcid.org/0000-0003-1995-8721

Universidade Federal de Pernambuco, Brasil

E-mail: m.suzane.barbosa@gmail.com

Fabio Torres Cunha

ORCID: https://orcid.org/0000-0003-0238-7600

Faculdade de Comunicação, Tecnologia e Turismo de Olinda, Brasil

E-mail: fabiologo1980@gmail.com

Silvia Mariana da Silva Barbosa

ORCID: https://orcid.org/0000-0001-5252-9705

Universidade Federal de Pernambuco, Brasil

E-mail: barbosasms@gmail.com

Danielle Cássia de Oliveira

ORCID: https://orcid.org/0000-0003-4154-2597

Faculdade de Comunicação, Tecnologia e Turismo de Olinda, Brasil

E-mail: daniellecassiao@yahoo.com.br

\begin{abstract}
Resumo
Objetivo: Investigar a relação da vigorexia com a insatisfação corporal, transtornos mentais e alimentares em adolescentes, adultos, levantadores de peso, frequentadores de academia e atletas do fisiculturismo. Metodologia: Trata-se de uma revisão bibliográfica efetuada no período de 2010 a 2020 com base nos dados das plataformas PubMed e Scielo. Resultados: Verificou-se 22 artigos inclusos e 12 exclusos, totalmente avaliados com base nos diversos fatores que influenciam no desenvolvimento da dismorfia muscular como o padrão de beleza posto pela sociedade e mídia que propagam informações e imagens de um corpo perfeito. Considerações Finais: Decorrente disso é essencial acompanhar os sinais mostrados pelo paciente e como conduzi-lo a priorizar o seu bem-estar físico e mental para que ocorra uma intervenção apropriada e uma orientação clínica-nutricional e psicológica adequada.
\end{abstract}

Palavras-chave: Transtorno mental; Comportamento alimentar; Anabolizantes; Dieta.

\begin{abstract}
Objective: To investigate the relationship between vigorexia and body dissatisfaction, mental and eating disorders in adolescents, adults, weightlifters, gym regulars and bodybuilding athletes. Methodology: This is a literature review conducted from 2010 to 2020 based on data from the PubMed and Scielo platforms.Results: It was verified that 22 articles were included and 12 were excluded, totally evaluated based on the several factors that influence the development of muscular dysmorphia, such as the standard of beauty set by society and the media that propagate information and images of a perfect body.Final Considerations: As a result, it is essential to follow the signs shown by the patient and how to lead them to prioritize their physical and mental well-being so that appropriate intervention and adequate clinical-nutritional and psychological guidance can occur.
\end{abstract}

Keyword: Mental disorder; Behavior eating; Anabolic steroids; Diet.

\section{Resumen}

Objetivo: Investigar la relación entre vigorexia e insatisfacción corporal, trastornos mentales y alimentarios en adolescentes, adultos, levantadores de pesas, asistentes al gimnasio y deportistas de musculación. Metodología: Se trata de una revisión bibliográfica realizada en el período de 2010 a 2020 a partir de datos de las plataformas PubMed y Scielo. Resultados: Se incluyeron 22 artículos y 12 se excluyeron, evaluados íntegramente en base a los diversos factores que influyen en el desarrollo de la dismorfia muscular como el estándar de belleza fijado por la sociedad y los 
medios de comunicación que propagan información e imágenes de un cuerpo perfecto.Consideraciones finales: En consecuencia, es fundamental monitorear las señales que muestra el paciente y cómo llevarlo a priorizar su bienestar físico y mental para que se produzca una intervención apropiada y una orientación clínico-nutricional y psicológica adecuada.

Palabras clave: Trastorno mental; Comportamiento alimenticio; Anabolizantes; Dieta.

\section{Introdução}

É eminente que a aplicação de exercícios físicos na rotina de um indivíduo oferece vantagens fisiológicas, psicológicas e sociais contribuindo para a precaução de patologias, aperfeiçoamento do metabolismo e força muscular, entretanto pode ser um motivo para surgir os transtornos associados ao corpo. Decorrente disso, o descontentamento dos indivíduos em relação à estética pode progredir ao quadro de transtorno mental e alimentar pela busca do corpo perfeito, podendo desenvolver a vigorexia (Bezerra et al., 2018).

O desenvolvimento da vigorexia também conhecida como dismorfia muscular ou anorexia reversa aumentou nas últimas décadas, obteve-se um aumento referente ao sexo masculino com idades entre 19 a 36 anos, onde buscam a perfeição corporal através da compulsão em se exercitar principalmente no levantamento de peso se baseando em uma dieta restritiva (Figueroa et al., 2018).

No quadro social e histórico da população, cada uma possui seus próprios padrões de beleza, moldadas e elaboradas relacionadas à concepção e percepção da sua respectiva imagem, formato e atividade física. No mundo Ocidental foi elaborado um ideal corporal (IDC) que leva tempo para atingir o objetivo do corpo perfeito devido os fatores materiais e psicossociais da modernidade, a imposição da sociedade para atingir o ideal corporal está associado à magreza para as mulheres e músculos para homens (Valenzuela et al., 2018).

A dieta é uma questão muito significativa na vigorexia, onde normalmente é restrita, com poucas variedades e quantidades elevadas, onde tendem a aumentar os macronutrientes carboidratos e principalmente proteínas diminuindo o consumo de lipídios além de suplementar proteína e fazer consumo de substâncias ergogênicas (Martinez et al., 2015).

Estudos mostraram que a atenção com a alimentação, peso e forma física varia entre etnias, e as mesmas estão relacionadas a figura corpórea, por exemplo: homens do continente da Ásia e hispânicos/latinos estão mais propensos a ter algum tipo de patologia comparado aos homens europeus e afro-americanos, relatando também que a análise corporal e preocupação com a muscularidade foi mais intensa em homens asiáticos, sendo necessária uma abordagem individualizada para esses indivíduos (Ramos et al., 2016).

A insatisfação do corpo no ser humano leva ao distúrbio dismórfico muscular, também denominado vigorexia, anorexia reversa ou complexo de Adonis os quais definem uma malformação anatômica, à medida que o termo psicopatológico é adequado para definir esse transtorno que está relacionado aos distúrbios alimentares (Ramos et al., 2016).

A distorção da figura do corpo é demonstrada em indivíduos que possuem uma rotina extremamente rígida, são fiéis com a verificação de cálculos do valor nutricional dos alimentos e diretrizes alimentares, intensos exercícios físicos e priorizam o alto consumo de proteínas. Outras ferramentas que levam ao diagnóstico da dismorfia muscular nos indivíduos é a retirada ou diminuição grave dos alimentos não protéicos, interrupção de atividades livres de interação social, ingerir alimentos constantemente em cerca de 2 ou 3 horas durante o dia, dão preferência a alimentação calórica líquida ou misturada na forma pastosa para facilitar a ingestão, restringem carboidratos e lipídios da dieta e abusam da proteína tanto na forma de alimento como em suplementos e anabolizantes que podem agravar esse transtorno levando ao quadro de doença renal crônica de acordo com (Behar \& Arancibia 2015). 
Este trabalho pretende elevar a importância de aumentar a atenção concedendo mais estudos relacionados à dismorfia muscular, tendo em vista que, é um diagnóstico recente na sociedade e que os níveis do mesmo nos indivíduos jovens estão aumentando, ocasionando preocupação aos profissionais da saúde.

Em virtude dos aspectos analisados, nota-se com este trabalho os comportamentos relacionados aos indivíduos que almejam mudança corporal acometidos pelo transtorno psicológico e alimentar. Assim, o presente estudo tem o objetivo de analisar a vigorexia em jovens e adultos de ambos os gêneros.

\section{Metodologia}

O presente trabalho é definido como uma revisão bibliográfica, quantitativa de cunho descritivo referente a vigorexia em acadêmicos, baseado em artigos mediante três idiomas: português, inglês e espanhol. Para a procura de artigos foram efetuadas pesquisas nas bases de dados como: Literature Analysis and Retrieval System online - PubMed (Medline) e Scientific Electronic Library Online (Scielo) no período de 2010 a 2020 com as consecutivas palavras-chave: transtorno mental, comportamento, anabolizantes e dieta.

Foram incluídos artigos indexados nas plataformas científicas de língua portuguesa, inglesa e espanhola, revisões de literatura, capítulo de livro, teses de mestrado e doutorado, estudos experimentais e sendo excluídas citações, tcc e arquivos duplicados. Os termos das palavras-chave foram selecionados e confirmados mediante a plataforma dos Descritores em Ciências da Saúde (DeCS). A busca consiste nas principais informações que consistem na frequência da vigorexia nos gêneros masculino e feminino na sociedade moderna.

\section{Resultados}

O resultado da busca nas bases de dados, descritos no fluxograma da Figura 1, resultou em: trinta e quatro (34) artigos no total. Dos 34 artigos iniciais, 12 foram excluídos da análise por não atenderem aos critérios pré-estabelecidos. Dos artigos incluídos restantes, 15 foram extraídos das bases de dados da Pubmed e 07 artigos foram extraídos das bases de dados do Scielo. Deste modo, vinte e dois artigos (22) integram esta revisão.

Desta forma após identificação dos artigos que cumpriram os critérios para inclusão, todos foram analisados e em seguida realizada a extração dos dados para a composição deste estudo. (Vide Tabela 1).

Figura 1. Fluxograma da pesquisa dos artigos.

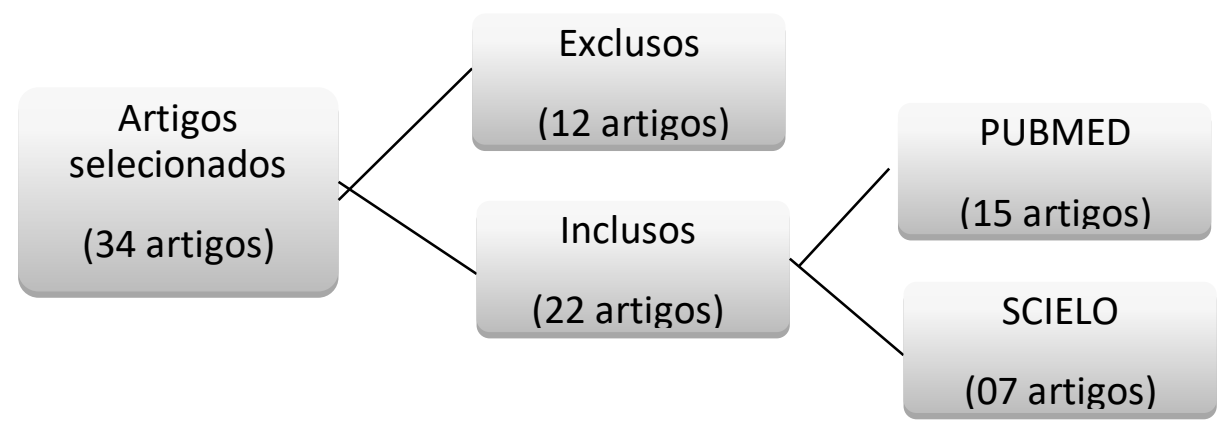

Fonte: Autores. 
Tabela 1. Objetivo, resultado e conclusão dos autores reunidos no presente estudo.

\begin{tabular}{llll}
\hline Autor/Ano & Objetivo & Resultados & Conclusão \\
\hline & Verificar a relação entre & Os adolescentes são um grupo & $\begin{array}{l}\text { A adolescência é um período da vida } \\
\text { de grandes mudanças, o indivíduo }\end{array}$ \\
mídia e escolhas & nutricional vulnerável, suas & $\begin{array}{l}\text { constrói a identidade corporal, busca } \\
\text { BITTAR, et al; 2020 }\end{array}$ \\
$\begin{array}{l}\text { alimentares na imagem } \\
\text { corporal e no possível }\end{array}$ & $\begin{array}{l}\text { necessidades nutricionais aumentam, } \\
\text { seudrão alimentar e estilo de vida }\end{array}$ & $\begin{array}{l}\text { referências corporais e a mídia causa } \\
\text { desenvolvimento de } \\
\text { transtornos alimentares }\end{array}$ \\
& em adem ser afetados por fatores & sentimento de insatisfação corporal por \\
internos e externos. & & propagar um padrão estético
\end{tabular}

Avaliar a associação entre a insatisfação e ARAÚJO, et al; imagem corporal entre 2019 homens e mulheres.

BADENES, et al; 2019

CLAUMANN, et al; 2019

JANKAUSKIENE, et al; 2019

LARISON, et al; 2019

Sintetizar as evidências científicas

disponíveis sobre a

ligação entre dismorfia

muscular

$\mathrm{e}$

sintomatologia do

transtorno alimentar.

O objetivo deste estudo foi estimar a prevalência de insatisfação com a imagem corporal, a associação entre a insatisfação pela magreza e pelo excesso de peso e componentes da aptidão física relacionada à saúde em adolescentes.

Avaliar a imagem corporal e determinantes relacionados ao esporte de atitudes e comportamentos alimentares (DEABs) perturbados na amostra de adolescente.

Explorar as contribuições relativas da auto-culpa e da vergonha internalizada à
A insatisfação da imagem corporal por excesso de peso prevalece nas mulheres $(57,3 \%$ vs. $30,6 \%$ nos homens) e uma maior insatisfação por défice nos homens $(27,8 \%$ vs. 9,3\% nas mulheres). variabilidade na alimentação desordenada e à busca de escores de muscularidade em homens colegiados.
Análise encontrou uma associação positiva entre os sintomas de dismorfia muscular e sintomatologia do transtorno alimentar. Mostraram que o tipo de amostra e as ferramentas para avaliar estavam estatisticamente associadas aos tamanhos de efeito delas juntas.

Ambos os sexos com adiposidade corporal elevada apresentaram menor chance de insatisfação pela magreza e maior chance de insatisfação pelo excesso de peso. Os que necessitavam melhorar a força muscular tiveram maior chance de insatisfação pela magreza.

Os DEABs, orientação da aparência, preocupação com excesso de peso e peso autoclassificado foram maiores nas meninas e os comportamentos de desenvolvimento da muscularidade nos meninos que praticam esportes competitivos.

A vergonha internalizada foi a única que contribuiu para a propulsão por muscularidade, a auto-culpa e a vergonha internalizada contribuíram para atitudes e comportamentos alimentares desordenados nos homens.
O consumo alimentar e o sexo dos indivíduos são variáveis modificadoras para aceitação da imagem corporal, sendo os homens o público mais satisfeito.

Níveis mais elevados de dismorfia muscular foram relacionados à maior sintomatologia da sintomatologia do transtorno alimentar.

Cerca de 3/4 da amostra investigada estava insatisfeita com sua imagem corporal. As associações encontradas entre imagem corporal e os componentes da aptidão física relacionada à saúde foram semelhantes entre os sexos.

Maiores preocupações com a imagem corporal e DEABs foram mais prevalentes em meninas que participam de esportes de lazer sensíveis ao peso, e a motivação para a muscularidade foi maior em meninos que participam de esportes de lazer sensíveis ao peso.

Tanto a culpa quanto a vergonha internalizada podem contribuir para a desordem alimentar nos homens. Além disso, a vergonha internalizada parece relacionar-se à busca de muscularidade nos homens. 


\section{Determinar a relação entre distúrbios da DEVRIM, et al; imagem corporal e 2018 \\ distúrbios alimentares em 120 fisiculturistas do sexo masculino.}

Avaliar a relação entre a insatisfação da imagem corporal, atitudes para KESSLER, et al; transtornos alimentares e 2018 o estado nutricional em universitárias da área da saúde.

Identificar a frequência do uso de esteroides OLIVEIRA, et al; anabolizantes por 2018 praticantes de musculação e os motivos que acarretaram o uso dessas substâncias e fazer associação com fatores sociodemográficos dos usuários.

Realizar uma revisão sistemática dos estudos

GARCÍA, et al; que, durante a última 2017 década, analisaram a relação entre dismorfia muscular e uso de substância ergogênica (ESU).

Analisar o nível e distribuição de insatisfação corporal em MEDEIROS, et al; mulheres que frequentam 2017 academia e quais fatores associados.

Detectar sintomas de dismorfia muscular em levantadores de peso masculinos, como as

HERNÁNDEZ, et características e al; 2017 comportamentos relacionados.

Comparar os níveis de insatisfação corporal, OLIVEIRA, et al; checagem corporal e 2017 comportamentos de risco para transtornos alimentares entre graduandas de cursos da área da saúde.

Os dados mostraram que quanto mais massa muscular adquirida, maior o comportamento para atingir mais muscularidade.

Ao relacionar a insatisfação da imagem corporal e atitudes de risco para transtornos alimentares, percebeu-se que $87,75 \%$ das universitárias com EAT positivo também apresentaram algum grau de insatisfação corporal.

Evidenciou-se que há um risco cerca de duas vezes maior para o uso de esteróides entre aqueles sujeitos com mais de um ano de prática de musculação, quando comparados com aqueles com menos tempo de prática.

Os resultados indicaram que a ESU não responde inequivocamente à presença de sintomas de dismorfia muscular, ou pelo menos não quando isso é avaliado pela combinação de atitudes e comportamentos.

A estética foi o principal motivo para as mulheres $(63,7 \%)$ se exercitarem e $21,4 \%$ retratavam insatisfação com o corpo.

Os dados relatam que $34,4 \%$ disseram que estavam satisfeitos com a sua muscularidade, com $59,4 \%$ expressaram insatisfação leve e $6,3 \%$ relataram que estavam moderadamente insatisfeitos com a sua muscularidade, que os revelou sintomas de dismorfia muscular e população em risco.

Os testes de comparação para as variáveis insatisfação, checagem corporal e comportamentos de risco para transtornos alimentares não demonstraram diferenças entre os grupos analisados.
Homens com distúrbios alimentares desejam ter uma forma mais muscular, principalmente em esportes relacionados ao peso corporal, como musculação, luta livre e levantamento de peso.

Evidenciou-se a existência de relação entre a insatisfação com a imagem corporal, atitudes de risco para transtornos alimentares e estado nutricional de universitárias da área da saúde.

O uso descontrolado dos esteróides anabolizantes por praticantes de musculação deixa claro que a vontade de conquistar o corpo ideal é preferenciada, ainda que sejam conhecidos os riscos dos efeitos colaterais.

Necessita-se trabalhar na formulação de estratégias que não apenas busquem influenciar a promoção de uma imagem corporal, conscientizando dos riscos que, a médio e longo prazo, podem levar a o uso e, acima de tudo, abuso de ergogênicos, prevenindo a dismorfia muscular.

Apesar do estudo não ter apresentado valores significativos, $(21,4 \%)$, as informações oferecidas por profissionais da área da saúde são necessárias para cessar o modismo dito pela indústria da beleza.

Os atletas podem apresentar sintomas de dismorfia muscular ao se envolver em uma atividade em que o desenvolvimento muscular é um fator determinante onde alguns participantes usavam substâncias para aumentar sua muscularidade e demonstravam comportamentos excessivos de verificação muscular.

Os estudos demonstram não haver diferenças significativas para a tais variáveis nutricionais dos cursos analisados -Medicina, Educação Física, Nutrição, Fisioterapia e Farmácia. 
Determinar os fatores associados ao consumo de

NABUCO, et al; suplementos alimentares 2016

em atletas brasileiros.

\section{6}

WIECKZOREK amor pela relaçáón

Body Image' (ABI) usando o modelo de componentes de dependência de Griffiths (2005) como estrutura para definir a vigorexia como um vício.

Investigar a relação entre risco de distúrbios

MAYO, et al; 2014 alimentares, insatisfação corporal e atratividade perceptiva em estudantes universitários do sexo masculino.

Identificar estratégias alimentares para

CONTESINI, et al; indivíduos fisicamente 2013

ativos com dismorfia muscular com base em uma revisão sistemática da literatura

Comparar os níveis de vigorexia e de dependência SOLER, et al; frequentadores encio entre $2013 \quad$ academias e fisiculturistas.

Examinar a insatisfação corporal e sua relação com a MAKINEN, et al; massa corporal, bem como a 2012
$\mathrm{Na}$ análise bivariada, sexo masculino, idade entre 25 e 29 anos, atividade remunerada, consumo de substâncias farmacológicas, distúrbio de imagem corporal, risco de dismorfia muscular, atletas com modalidade de potência e falta de assistência médica foram fatores associados à ingestão de suplementos.

Houve excesso de preocupação com o teor de massa gorda corporal e volume da musculatura e abdicação das atividades livres sociáveis para permanecer a rotina de treinos $\mathrm{e}$ dietas.

Perceberam que a atividade viciante na vigorexia é a manutenção da imagem corporal por meio exercícios, comer certos alimentos e tomar medicamentos. No modelo $\mathrm{ABI}$, a percepção dos efeitos na auto-imagem corporal é considerada um aspecto crítico.

Houve correlação positiva entre o Teste de Atitudes Alimentares (EAT) e a insatisfação gordurosa e correlação negativa para a insatisfação muscular, $28 \%$ dos homens fizeram um EAT pontuação indicando que eles estavam em risco de um distúrbio alimentar.

Indicaram que a dieta incluía alimentos ricos em proteínas e baixo teor de gordura e a ingestão de suplementos alimentares e ergogênicos para reduzir o peso sem acompanhamento de nutricionista.

Verificou-se que o grupo classificado como dependente de exercícios físicos revela níveis médios superiores de vigorexia.

As meninas e os meninos manifestaram maior satisfação com o corpo quando estavam abaixo do peso, mais insatisfação quando tinham peso normal e mais insatisfação quando tinham excesso de peso.
É necessário o trabalho de profissionais em estreita colaboração com os atletas para monitorar continuamente o uso de suplementos, uma vez que se trata de um comportamento alimentar associado a outro comportamento de risco (uso farmacológico), bem como a distúrbios de imagem (dismorfia muscular).

Nota-se a importância de atender as pessoas difíceis de haver comunicação, seu comportamento através do discurso fazendo necessário problematizar e trazer contribuições do reconhecimento da complexidade do psiquismo do ser humano.

É apontado que a vigorexia poderia ser reclassificada como um vício, devido aos comportamentos repetitivos dos indivíduos em relação a imagem corporal, o que podem causar danos a longo prazo.

Homens em idade universitária podem estar em risco de distúrbios alimentares com base em distorções na imagem corporal ideal percebida, tanto para gordura quanto para músculo.

Indivíduos fisicamente ativos com dismorfia muscular poderiam se beneficiar da ajuda de profissionais da nutrição para avaliar a estimativa de energia, orientar a dieta e sua distribuição em macronutrientes.

Por fim, constatou-se que tanto nos fisiculturistas como nos frequentadores de academias, quanto maior o nível de vigorexia, maior o nível de dependência ao exercício, sendo essa correlação maior em fisiculturistas.

A massa corporal, a autoestima e os hábitos alimentares revelaram uma relação significativa com a insatisfação corporal na fase de transição do início ao meio da adolescência em meninas e meninos, mas também foram encontradas diferenças significativas entre os sexos. 


$\begin{array}{lll}\text { Verificar os motivos e a } & \text { Verificou-se que a estética, a saúde } \\ \text { prevalência de insatisfação } & \text { e outros são os motivos que mais } \\ \text { com a imagem corporal (IC) } & \begin{array}{l}\text { influenciam a insatisfação com a } \\ \text { em adolescentes. }\end{array}\end{array}$

Avaliar o exercício físico, BEHAR, et al; comportamentos

2010 alimentares e presença de dismorfia muscular entre levantadores de peso e estudantes de medicina.

\begin{abstract}
Os levantadores de peso gastaram mais tempo do que os alunos que se exercitam para melhorar sua aparência. $42 \%$ dos levantadores de peso com dismorfia muscular apresentaram abuso de anabolizantes e $67 \%$ usaram outras substâncias para melhorar seu desempenho.
\end{abstract}

Mais da metade dos adolescentes está insatisfeita com sua silhueta corporal. Neste sentido, intervenções por profissionais e serviços de saúde são necessárias nessa fase da vida, evitando possíveis problemas futuros de distúrbios alimentares (anemia, bulimia e vigorexia).

A presença de dismorfia muscular entre os levantadores de peso foi confirmada. Eles estavam insatisfeitos com a imagem corporal e mais preocupados com a aparência física do que aqueles sem dismorfia muscular e/ou estudantes.

Fonte: Autores.

\section{Discussão}

Segundo Bittar e Soares (2020), encontraram que os adolescentes são um grupo nutricionalmente vulnerável, onde a Organização Mundial da Saúde determina que nesse período da adolescência a atenção deve ser priorizada pois suas necessidades nutricionais aumentam, seu padrão alimentar e estilo de vida podem ser afetados por diversos fatores internos como a formação da autoimagem e externos como a sociedade contemporânea onde a mídia que estabelece alimentação e padrões de beleza.

Já Petroski et al. (2012) obteve resultados semelhantes a Bittar em relação a alta prevalência de insatisfação com a imagem corporal dos adolescentes, as moças apresentaram maior insatisfação corporal com $65,7 \%$ do que os rapazes com $54,5 \%$. Foi observado que a estética e a autoestima são os motivos que mais influenciam na distorção da imagem corporal em adolescentes, sendo a estética contendo 53,7\% influenciando a distorção da imagem corporal nos adolescentes urbanos, enquanto que a baixa autoestima com 50,9\% como o motivo que mais distorce a imagem corporal nos adolescentes rurais.

A insatisfação da imagem corporal por excesso de peso prevalece nas mulheres (57,3\% vs. 30,6\% nos homens) e uma maior insatisfação por défice nos homens ( $27,8 \%$ vs. 9,3\% nas mulheres) os quais manifestaram o desejo de aumentar o seu tamanho corporal. Nas mulheres, a insatisfação com a imagem corporal apresentou uma maior ingestão de gordura poliinsaturada, enquanto os homens obtiveram um menor consumo de alimentos densamente energéticos, como relatam Araújo e Oliveira (2019).

Já em outro estudo de Larison e Pritchard (2019) retratam que os homens possuem níveis mais altos de auto-culpa e vergonha internalizada devido a insatisfação corporal. Prevalecendo a vergonha internalizada como desencadeadora para a propulsão por muscularidade e para atitudes e comportamentos alimentares desordenados nos homens ocorrendo sintomatologia do transtorno alimentar.

Também foi encontrada relações entre os sintomas de dismorfia muscular (MD) e sintomatologia do transtorno alimentar (ED) relatando que o tipo de amostra e as ferramentas para avaliação estavam estatisticamente associadas aos tamanhos de efeito de ambas (MD-ED) onde obtiveram associações mais fortes quando foi realizado entre pessoas que usavam esteróides anabolizantes (AAS) em comparação com outras amostras de acordo com uma análise feita por Badenes et al. (2019). 
Comparando à análise de García et al. (2017) também baseada no assunto de dismorfia muscular, os resultados indicaram que embora os sintomas da mesma estão mais presentes nos levantadores de peso como fisiculturistas não naturais, indicando que o uso de substâncias ergogênicas não respondem inequivocamente à presença de sintomas de dismorfia muscular, ou pelo menos não quando isso é avaliado pela combinação de atitudes e comportamentos.

Tanto o sexo masculino como o feminino com adiposidade corporal elevada apresentaram menor chance de insatisfação pela magreza e maior chance de insatisfação pelo excesso de peso. De acordo com Claumann et al (2019) a prevalência de insatisfação com a imagem corporal na amostra resultou em 75,2\%, sendo que 39,5 dos adolescentes estavam insatisfeitos com excesso de peso e 35,7 pela magreza e os que necessitavam melhorar a força muscular tiveram maior chance de insatisfação pela magreza.

Segundo Makinen et al. (2012) demonstrou que o sexo feminino apresentava maiores níveis de depressão em relação a insatisfação corporal e menores de autoestima do que o sexo masculino. Verificou-se que ambos os sexos com $32.2 \%$ estavam satisfeitos com sua imagem corporal, 44,6\% gostariam de diminuir a silhueta e 23,2\% gostariam de aumentar. Com base nos dados, a insatisfação corporal e depressão foi maior com as que auto avaliaram negativamente a saúde e consumo de bebidas alcoólicas e menor entre as adolescentes eutróficas e aquelas que praticavam atividade física, verificou-se que as mais ativas possuíam menor depressão.

Indivíduos que possuem dismorfia muscular têm seu comportamento relacionado a causas distintas, motivos individuais e sociais levam a mudanças psicológicas que contribuem para o progresso da patologia e um fator considerável, é a insatisfação e alteração da imagem física pelo padrão de beleza instigado pela pressão da sociedade e mídia como relata Foster et al. (2015).

Na concepção de Wieckzorek, (2016) esse distúrbio é fundamentado nas circunstâncias que o indivíduo possui com sua imagem corporal ser suficiente em nenhum momento e para isso o mesmo estabeleceu parâmetros mais ocorridos nessa patologia. Dos artigos incluídos (20) salientam que os parâmetros incluem a aflição expressiva com a preocupação do teor de massa gorda corporal e volume da musculatura, o intenso monitoramento de treino e dietas, abdicar as atividades livres sociáveis para permanecer a rotina de treinos e dietas, esquivar de episódios que envolvam a exibição física devido a não se agradar com o corpo.

Mayo e George (2014) compararam o Teste de Atitudes Alimentares (EAT) e insatisfação gordurosa os quais mostraram correlação positiva e correlação negativa para insatisfação muscular onde $28 \%$ dos homens apresentaram uma pontuação no EAT indicando que eles estavam em risco de um distúrbio alimentar. Os mesmos escolheram um padrão de corpo significativamente mais musculoso e mais magro do que o feminino optou por ser atraente.

Em outra análise de Cortesini et al. (2013) indicou que a maioria dos indivíduos fisicamente ativos em academias seguem dietas específicas com objetivo de ganhar massa muscular e perder gordura, mas ficou evidente nos resultados da pesquisa que a maioria prepara suas próprias dietas aleatoriamente. A dieta incluía alimentos ricos em proteínas e baixo teor de gordura e a ingestão de suplementos alimentares e ergogênicos para reduzir o peso, sem conhecimento ou orientação nutricional específica dos profissionais.

Os dados revelados por Devrim et al. (2018) demonstraram que quanto mais músculos os participantes ganhavam, mais eles trabalham para a muscularidade. Dos 120 fisiculturistas, 81 (67,5\%) apresentaram altos escores no Teste de Atitudes Alimentares (EAT), indicando níveis potencialmente clínicos de patologia alimentar, tanto os fisiculturistas com e sem transtornos alimentares relataram que planejam seus horários de dieta e cozinham para si mesmos. Essa análise indica que um distúrbio alimentar foi um fator de risco relativo para sintomas de dismorfia muscular e insatisfação corporal relacionada ao músculo. 
Por conseguinte, De Oliveira e Neto (2018) observaram que a melhoria da estética foi o principal motivo para que esses praticantes de musculação fizessem uso de esteróides anabolizantes, entretanto a maioria desses usuários possuíram efeitos colaterais (28\%), adquiriram tais produtos através de amigos (63\%) e não teve qualquer acompanhamento médico (97,8\%) para utilização dessa substância. Com base na estética há um risco cerca de duas vezes maior para o uso de esteróides entre aqueles sujeitos com mais de um ano de prática de musculação, quando comparados com aqueles com menos tempo de prática.

Os levantadores de pesos insatisfeitos com sua muscularidade, ingerem algum tipo de suplemento para aumentar o seu peso feito diariamente ou duas vezes por semana, onde essas substâncias são shakes de carboidratos e proteínas nas quantidades recomendadas conforme demonstra Hernández et al. (2017). Esses que estão insatisfeitos gastam mais com substâncias e que também experimentam qualquer tipo de produto para aumentar sua muscularidade, também se olham no espelho para verificar qualquer alteração na aparência física sempre que possível.

Outro estudo relata resultados semelhantes onde o interesse na aparência física foi significativamente maior entre os levantadores de peso que possuíam dismorfia muscular queriam aumentar sua figura, direcionavam o peso mais semanalmente e olhavam mais no espelho em comparação aos que não apresentavam dismorfia e estudantes como mencionam Behar e Molinari (2010). Com isso, os levantadores de peso gastaram mais tempo do que os alunos que se exercitam para melhorar sua aparência. $42 \%$ dos levantadores de peso com dismorfia muscular apresentaram abuso de anabolizantes e $67 \%$ usaram outras substâncias para melhorar seu desempenho.

Kessler e Poll (2018) relacionam a insatisfação da imagem corporal e atitudes de risco para transtornos alimentares, perceberam que 87,75\% das universitárias com Teste de Atitudes Alimentares (EAT) positivo também apresentaram algum grau de insatisfação corporal. Pois, quando o mesmo fez um cruzamento das classificações do Índice de Massa Corpórea (IMC) e Teste de Atitudes Alimentares (EAT), a pontuação positiva do teste foi mais prevalente em universitárias com estado de eutrofia. Entretanto, juntamente com as classificações para sobrepeso, obesidade grau I e obesidade grau II, os dados revelaram semelhantes aos dos eutróficos.

Contrapondo a pesquisa de Oliveira et al. (2017) onde contou com dados de 92 estudantes analisados e a partir do teste de comparações entre os grupos para as variáveis analisadas, foram reveladas diferenças estatísticas apenas para a idade entre estudantes de Educação Física e Medicina, sendo este último grupo o mais jovem. Os testes de comparação para as variáveis insatisfação corporal, checagem corporal e comportamentos de risco para transtornos alimentares não demonstraram diferenças entre os grupos analisados.

Jankauskiene e Baceviciene (2019) encontraram uma forte interação de gênero no grupo de sensibilidade ao peso. As mulheres que praticam esportes de lazer sensíveis ao peso apresentavam comportamentos alimentares desordenados e comprometedores da saúde e homens relataram um desejo maior de muscularidade, quando comparado com ambos gêneros participantes de esportes de lazer menos sensíveis ao peso. Assim, prevalece a maior preocupação entre as mulheres que participam de esportes de lazer sensíveis ao peso com excesso de peso em comparação com aquelas que participam de esportes de lazer com menos peso.

Verificando com a análise de Medeiros et al. (2017) a qual também mostra a preocupação das mulheres com o peso, relatou que 67,3\% das mesmas afirmaram que um dos motivos que as levavam a praticar exercícios está a estética, e 63,8\% assumiram que poderiam realizar cirurgia plástica. Ao analisar a distribuição de insatisfação corporal encontram associadas ao desfecho as variáveis práticas de exercícios por motivos estéticos, Índice de Massa Corpórea (IMC), autopercepção de saúde, desejo de realizar cirurgia plástica e qualidade de vida.

De acordo com o estudo de Nabuco et al. (2016) atletas que tomaram suplementos alimentares usaram mais substâncias farmacológicas, todos os usuários de esteroides anabolizantes e redutores de gordura tomaram suplementos 
alimentares. Em relação aos usuários de estimulantes e diuréticos, $93 \%$ e 72,7\%, respectivamente, também tomaram suplementos. Não houve associação entre uso de suplementos e Índice de Massa Corpórea (IMC), escolaridade, consumo de álcool ou fase de treinamento.

Já em relação a (Soler et al. (2013) sua análise relata que o tempo de prática de exercício físico está negativamente associado aos efeitos intencionais e à intolerância com a aparência. A duração semanal do tempo de treino foi a variável com maior efeito correspondente tendo-se associação positiva à maioria das dimensões da dependência ao exercício (abstinência, a continuidade, a perda de controle, o tempo e os efeitos intencionais).

\section{Considerações Finais}

A presente revisão bibliográfica sugere que ambos os gêneros (masculino e feminino) se encontram insatisfeitos com a sua imagem corporal, onde possuem associação com o consumo alimentar sendo mais acometido em homens e praticantes de esportes do fisiculturismo, todavia também afeta as mulheres que há séculos são exigidas a seguir um padrão de beleza associado à magreza. Na elaboração da revisão bibliográfica a dificuldade está relacionada a quantidade de artigos, visto que possuem poucos relacionados a vigorexia devido ser um diagnóstico recente na sociedade.

Portanto, ressalta a importância de uma orientação clínica adequada com profissionais capacitados como nutricionistas aliados aos psicólogos para correção de danos à saúde fisiológica e mental, através de transmissão e práticas de conhecimentos sobre dietas equilibradas e a autopercepção da imagem corporal. Todavia, ainda é necessário a condução de estudos longitudinais futuros.

\section{Referências}

Araújo, A. C., \& Oliveira, A. (2019). (In) Satisfação com a imagem corporal: associação com o consumo alimentar e a ingestão nutricional. Acta Portuguesa de Nutrição, (16), 18-24. http://dx.doi.org/10.21011/apn.2019.1604.

Badenes-Ribera, L., Rubio-Aparicio, M., Sánchez-Meca, J., Fabris, M. A., \& Longobardi, C. (2019). The association between muscle dysmorphia and eating disorder symptomatology: A systematic review and meta-analysis. Journal of behavioral addictions, 8(3), 351-371. https://doi.org/10.1556/2006.8.2019.44.

Behar, R., \& Arancibia, M. (2015). Trastornos de la imagen corporal: Anorexia nerviosa versus anorexia inversa (trastorno dismórfico muscular). Revista mexicana de trastornos alimentarios, 6(2), 121-128. https://doi.org/10.22201/fesi.20071523e.2015.2.344.

Behar, R., \& Molinari, D. (2010). Dismorfia muscular, imagen corporal y conductas alimentarias en dos poblaciones masculinas. Revista médica de Chile, 138(11), 1386-1394. https://dx.doi.org/10.4067/S0034-98872010001200007.

Bezerra, D. F., Sampaio, L. V. A., \& Landim, L. A. D. S. R. (2018). Diagnóstico de vigorexia e dismorfia muscular em universitários da área da saúde. Nutrición clínica y dietética hospitalaria, 38(4), 179-182. http://doi.org/10.12873/384liejy.

Bittar, C., \& Soares, A. (2020). Mídia e comportamento alimentar na adolescência. Cadernos Brasileiros de Terapia Ocupacional, 28(1), 291-308. https://doi.org/10.4322/2526-8910.ctoar1920.

Claumann, G. S., Laus, M. F., Felden, É. P. G., Silva, D. A. S., \& Pelegrini, A. (2019). Associação entre insatisfação com a imagem corporal e aptidão física relacionada à saúde em adolescentes. Ciência \& Saúde Coletiva, 24, 1299-1308. https://doi.org/10.1590/1413-81232018244.17312017.

Contesini, N., Adami, F., Blake, M. D. T., Monteiro, C. B., Abreu, L. C., Valenti, V. E., \& Frainer, D. E. S. (2013). Nutritional strategies of physically active subjects with muscle dysmorphia. International archives of medicine, 6(1), 25. https://doi.org/10.1186/1755-7682-6-25.

De Oliveira, L. L., \& Neto, J. L. C. (2018). Fatores sociodemográficos, perfil dos usuários e motivação para o uso de esteroides anabolizantes entre jovens adultos. Revista Brasileira de Ciências do Esporte, 40(3), 309-317. https://doi.org/10.1016/j.rbce.2018.03.015.

Devrim, A., Bilgic, P., \& Hongu, N. (2018). Is there any relationship between body image perception, eating disorders, and muscle dysmorphic disorders in male bodybuilders? American journal of men's health, 12(5), 1746-1758. https://doi.org/10.1177/1557988318786868.

Figueroa, M. A. F., Troya, T. C., López, S. J., González, G. A., \& Cruz, A. V. (2018). Estudio neuropsicológico de la dismorfia muscular y del trastorno obsesivo-compulsivo. Universitas Psychologica, 17(3), 1-10. https://doi.org/10.11144/Javeriana.upsy 17-3.endm.

Foster, A., Shorter, G., \& Griffiths, M. (2015). Muscle dysmorphia: Could it be classified as an addiction to body image?. Journal of Behavioral Addictions, 4(1), 1-5. https://doi.org/10.1556/jba.3.2014.001.

García-Rodríguez, J., et al. "Muscle dysmorphia and use of ergogenics substances. A systematic review." Revista Colombiana de Psiquiatría (English ed.) 46.3 (2017): 168-177. https://doi.org/10.1016/j.rcpeng.2017.06.003. 
Research, Society and Development, v. 10, n. 5, e16710514817, 2021

(CC BY 4.0) | ISSN 2525-3409 | DOI: http://dx.doi.org/10.33448/rsd-v10i5.14817

Hernández-Martínez, A., Irene, G.-M., \& Onofre, R C. J. "Detection of Muscle Dysmorphia symptoms in male weightlifters." Anales de Psicología/Annals of Psychology 33.1 (2017): 204-210. https://doi.org/10.6018/analesps.

Jankauskiene, R., \& Miglè, B. "Body image and disturbed eating attitudes and behaviors in sport-involved adolescents: the role of gender and sport characteristics." Nutrients 11.12 (2019): 3061. https://doi.org/10.3390/nu11123061.

Kessler, A. L., \& Fabiana, A. P. "Relação entre imagem corporal, atitudes para transtornos alimentares e estado nutricional em universitárias da área da saúde." Jornal Brasileiro de Psiquiatria 67.2 (2018): 118-125. https://doi.org/10.1590/0047-2085000000194.

Larison, B., \& Mary, P. "The effects of internalized shame and self-blame on disordered eating and drive for muscularity in collegiate men." Eating and Weight Disorders-Studies on Anorexia, Bulimia and Obesity 24.4 (2019): 653-660. https://doi.org/10.1007/s40519-019-00700-0.

Mäkinen, M., et al. "Body dissatisfaction and body mass in girls and boys transitioning from early to mid-adolescence: additional role of self-esteem and eating habits." BMC psychiatry 12.1 (2012): 1-8. https://doi.org/10.1186/1471-244X-12-35.

Martínez Segura, A., et al. "Valoración de la dieta de usuarios de sala de musculación con dismorfia muscular (vigorexia)." Nutrición Hospitalaria 32.1 (2015): 324-329. http://dx.doi.org/10.3305/nh.2015.32.1.8922.

Mayo, C., \& Valerie, G. "Eating disorder risk and body dissatisfaction based on muscularity and body fat in male university students." Journal of American College Health 62.6 (2014): 407-415. https://doi.org/10.1080/07448481.2014.917649.

Medeiros, T. H., Eduardo, L. C., \& Marlos, R. D.. "Insatisfação corporal em frequentadoras de academia." Jornal Brasileiro de Psiquiatria 66.1 (2017): $38-44$. http://dx.doi.org/10.1590/0047-2085000000148.

Nabuco, H. C. G., Rodrigues, V. B., Fernandes, V. L. S., de Paula Ravagnani, F. C., Fett, C. A., Espinosa, M. M., \& Ravagnani, C. D. F. C. (2016). Factors associated with dietary supplementation among Brazilian athletes. Nutrición Hospitalaria, 33(3), 678-684. 10.20960/nh.278.

Oliveira, P. L. D., Ferreira, M. E. C., Neves, C. M., Meireles, J. F. F., \& Carvalho, P. H. B. D. (2017). Insatisfação, checagem corporal e comportamentos de risco para transtornos alimentares em estudantes de cursos da saúde. Jornal Brasileiro de Psiquiatria, 66(4), 216-220. http://doi.org/10.1590/00472085000000174.

Petroski, E. L., Pelegrini, A., \& Glaner, M. F. (2012). Motivos e prevalência de insatisfação com a imagem corporal em adolescentes. Ciência \& Saúde Coletiva, 17(4), 1071-1077. https://doi.org/10.1590/S1413-81232012000400028.

Ramos, T. D., Brito, M. J. A. D., Piccolo, M. S., Rosella, M. F. N. D. S. M., Sabino Neto, M., \& Ferreira, L. M. (2016). Body Dysmorphic Symptoms Scale for patients seeking esthetic surgery: cross-cultural validation study. Sao Paulo Medical Journal, 134(6), 480-490. https://doi.org/10.1590/15163180.2016 .0068160416

Soler, P. T., Fernandes, H. M., Damasceno, V. O., \& Novaes, J. S. (2013). Vigorexia e níveis de dependência de exercício em frequentadores de academias e fisiculturistas. Revista brasileira de medicina do esporte, 19(5), 343-348. https://doi.org/10.1590/S1517-86922013000500009.

Valenzuela Gutiérrez, M. E., \& Meléndez Torres, J. M. (2018). Concepciones y valoraciones del cuerpo en jóvenes del noroeste de México. Revista mexicana de trastornos alimentarios, 9(1), 45-56. https://doi.org/10.22201/fesi.20071523e.2018.1.456.

Wieczorek, R. T. (2016). Da academia para o divã: reflexões sobre o narcisismo. Aletheia, 49(2). http://doi.org/10.29327/226091. 